\begin{tabular}{|c|c|}
\hline Title & Free hy perplane arrangements over arbitrary fields \\
\hline Author(s) & Torielli, Michele; Palezzato, Elisa \\
\hline Citation & $\begin{array}{l}\text { Journal of algebraic combinatorics, } 52(2), 237-249 \\
\text { https://doi.org/10.1007/s10801-019-00901-x }\end{array}$ \\
\hline Issue Date & $2020-09$ \\
\hline Doc URL & http:/hdl.handle.net/2115/82565 \\
\hline Rights & $\begin{array}{l}\text { This is a post-peer-review, pre copyedit version of an article published in Journal of A lgebraic Combinatorics. The final } \\
\text { authenticated version is available online at: } h t \text { ttp://dx.doi.org/10.1007/s10801-019-00901- } x^{\prime \prime} \text {. }\end{array}$ \\
\hline Type & article (author version) \\
\hline File Information & J. A lgebr. Comb.52-2_237-249.pdf \\
\hline
\end{tabular}

Instructions for use 


\title{
Free hyperplane arrangements over arbitrary fields
}

\author{
Michele Torielli • Elisa Palezzato
}

Received: date / Accepted: date

\begin{abstract}
In this paper, we study the class of free hyperplane arrangements. Specifically, we investigate the relations between freeness over a field of finite characteristic and freeness over $\mathbb{Q}$.
\end{abstract}

Keywords Free hyperplane arrangement · Cohen-Macaulay ideal · Logarithmic derivations

\section{Introduction}

Let $V$ be a vector space of dimension $l$ over a field $K$. Fix a system of coordinates $\left(x_{1}, \ldots, x_{l}\right)$ of $V^{*}$. We denote by $S=S\left(V^{*}\right)=K\left[x_{1}, \ldots, x_{l}\right]$ the symmetric algebra. A hyperplane arrangement $\mathcal{A}=\left\{H_{1}, \ldots, H_{n}\right\}$ is a finite collection of hyperplanes in $V$.

Freeness of an arrangement is a key notion which connects arrangement theory with algebraic geometry and combinatorics. The study of free arrangements was started by Saito [14] and a remarkable factorization theorem was proved by Terao [16]. This theorem asserts that the characteristic polynomial of a free arrangement completely factors into linear polynomials over the integers. This imposes a necessary condition on the structure of the intersection lattice for an arrangement to be free. The Terao conjecture is the converse problem, i.e. to understand if the structure of the intersection lattice characterize freeness of arrangements. A lot of work has been done to solve this conjecture especially in the case of characteristic 0 (see for example [18], [19], [3], [1] and [6]). However, also the case of finite characteristic

Michele Torielli

Department of Mathematics, Hokkaido University, Kita 10, Nishi 8, Kita-Ku, Sapporo 060-0810, Japan. E-mail: torielli@math.sci.hokudai.ac.jp

Elisa Palezzato

Department of Mathematics, Hokkaido University, Kita 10, Nishi 8, Kita-Ku, Sapporo 060-0810, Japan. E-mail: palezzato@math.sci.hokudai.ac.jp 
has been analyzed (see for example [17] and [20]). Despite this effort, this question is still open.

The purpose of this paper is to study the connections between freeness over a field of characteristic zero and over a finite field, and to describe in which cases the two situations are related and how.

\section{Preliminares on hyperplane arrangements}

In this section, we recall the terminology, the basic notations and some fundamental results related to hyperplane arrangements.

Let $K$ be a field. A finite set of affine hyperplanes $\mathcal{A}=\left\{H_{1}, \ldots, H_{n}\right\}$ in $K^{l}$ is called a hyperplane arrangement. For each hyperplane $H_{i}$ we fix a polynomial $\alpha_{i} \in S=K\left[x_{1}, \ldots, x_{l}\right]$ such that $H_{i}=\alpha_{i}^{-1}(0)$, and let $Q(\mathcal{A})=\prod_{i=1}^{n} \alpha_{i}$. An arrangement $\mathcal{A}$ is called central if each $H_{i}$ contains the origin of $K^{l}$. In this case, the defining polynomial $\alpha_{i} \in S$ is linear homogeneous, and hence $Q(\mathcal{A})$ is homogeneous of degree $n$.

Let $L(\mathcal{A})=\left\{\bigcap_{H \in \mathcal{B}} H \mid \mathcal{B} \subseteq \mathcal{A}\right\}$ be the lattice of intersection of $\mathcal{A}$, ordered by reverse inclusion, i.e. $X \leq Y$ if and only if $Y \subseteq X$, for $X, Y \in L(\mathcal{A})$. Define a rank function on $L(\mathcal{A})$ by $\operatorname{rk}(X)=\operatorname{codim}(X) . L(\mathcal{A})$ plays a fundamental role in the study of hyperplane arrangements, in fact it determines the combinatorics of the arrangement.

Let $\mu: L(\mathcal{A}) \longrightarrow \mathbb{Z}$ be the Möbius function of $L(\mathcal{A})$ defined by

$$
\mu(X)= \begin{cases}1 & \text { for } X=K^{l}, \\ -\sum_{Y<X} \mu(Y) & \text { if } X>K^{l} .\end{cases}
$$

The characteristic polynomial of $\mathcal{A}$ is defined by

$$
\chi(\mathcal{A}, t)=\sum_{X \in L(\mathcal{A})} \mu(X) t^{\operatorname{dim}(X)} .
$$

For $i=0, \ldots, l$ we define the $i$-th Betti number $b_{i}(\mathcal{A})$ by the formula

$$
\chi(\mathcal{A}, t)=\sum_{i=0}^{l}(-1)^{i} b_{i}(\mathcal{A}) t^{l-i} .
$$

The importance of the characteristic polynomial in combinatorics is justified by the following result from [7], [11] and [21].

Theorem 2.1 We have that

1. If $\mathcal{A}$ is an arrangement in $\mathbb{F}_{p}^{l}$, then $\left|\mathbb{F}_{p}^{l} \backslash \bigcup_{H \in \mathcal{A}} H\right|=\chi(\mathcal{A}, p)$.

2. If $\mathcal{A}$ is an arrangement in $\mathbb{C}^{l}$, then the topological $i$-th Betti number of the complement is $b_{i}\left(\mathbb{C}^{l} \backslash \bigcup_{H \in \mathcal{A}} H\right)=b_{i}(\mathcal{A})$.

3. If $\mathcal{A}$ is an arrangement in $\mathbb{R}^{l}$, then $|\chi(\mathcal{A},-1)|$ is the number of chambers and $|\chi(\mathcal{A}, 1)|$ is the number of bounded chambers. 


\section{Free hyperplane arrangements}

We recall the basic notions and properties of free hyperplane arrangements.

We denote by $\operatorname{Der}_{K^{l}}=\left\{\sum_{i=1}^{l} f_{i} \partial_{x_{i}} \mid f_{i} \in S\right\}$ the $S$-module of polynomial vector fields on $K^{l}$ (or $S$-derivations). Let $\delta=\sum_{i=1}^{l} f_{i} \partial_{x_{i}} \in \operatorname{Der}_{K^{l}}$. Then $\delta$ is said to be homogeneous of polynomial degree $d$ if $f_{1}, \ldots, f_{l}$ are homogeneous polynomials of degree $d$ in $S$. In this case, we write $\operatorname{pdeg}(\delta)=d$.

Definition 3.1 Let $\mathcal{A}$ be a central arrangement in $K^{l}$. Define the module of vector fields logarithmic tangent to $\mathcal{A}$ (or logarithmic vector fields) by

$$
D(\mathcal{A})=\left\{\delta \in \operatorname{Der}_{K^{l}} \mid \delta\left(\alpha_{i}\right) \in\left(\alpha_{i}\right) S, \forall i\right\} .
$$

The module $D(\mathcal{A})$ is obviously a graded $S$-module and we have that $D(\mathcal{A})=$ $\left\{\delta \in \operatorname{Der}_{K^{l}} \mid \delta(Q(\mathcal{A})) \in(Q(\mathcal{A})) S\right\}$. In particular, since the arrangement $\mathcal{A}$ is central, then the Euler vector field $\delta_{E}=\sum_{i=1}^{l} x_{i} \partial_{x_{i}}$ belongs to $D(\mathcal{A})$, in fact $\delta_{E}(Q(\mathcal{A}))=n Q(\mathcal{A})$.

Definition 3.2 A central arrangement $\mathcal{A}$ in $K^{l}$ is said to be free with exponents $\left(e_{1}, \ldots, e_{l}\right)$ if and only if $D(\mathcal{A})$ is a free $S$-module and there exists a basis $\delta_{1}, \ldots, \delta_{l}$ of $D(\mathcal{A})$ such that $\operatorname{pdeg}\left(\delta_{i}\right)=e_{i}$, or equivalently $D(\mathcal{A}) \cong \bigoplus_{i=1}^{l} S\left(-e_{i}\right)$.

In general the exponents of an arrangement depend on the characteristic of $K$. In fact, we have the following examples.

Example 3.3 ([12], Example 4.35) Consider the arrangement $\mathcal{A}$ in $K^{3}$ with defining polynomial $Q(\mathcal{A})=x y z(x-y)(x+z)(y+z)(x+y+z)$. Then $\mathcal{A}$ is free for any $K$, but its exponents depend on the characteristic of $K$.

If $\operatorname{char}(K) \neq 2$, then $\mathcal{A}$ is free with exponents $(1,3,3)$, in fact we can take as basis of $D(\mathcal{A})$ the following vector fields $\delta_{E}, \delta_{2}=x(x+z)(x+y+z) \partial_{x}+y(y+$ $z)(x+y+z) \partial_{y}$ and $\delta_{3}=x(x+z)(2 y+z) \partial_{x}+y(y+z)(2 x+z) \partial_{y}$.

If $\operatorname{char}(K)=2$, then $\mathcal{A}$ is free with exponents $(1,2,4)$, in fact we can take as basis of $D(\mathcal{A})$ the following vector fields $\delta_{E}, \delta_{2}=x^{2} \partial_{x}+y^{2} \partial_{y}+z^{2} \partial_{z}$ and $\delta_{3}=x^{4} \partial_{x}+y^{4} \partial_{y}+z^{4} \partial_{z}$

Example 3.4 Consider $\mathcal{A}$ the arrangement in $K^{3}$ as the cone of $\mathcal{A}^{[-2,2]}$ the ShiCatalan arrangement of type $B$, see for example [2]. Then $\mathcal{A}$ is free for any $K$, but its exponents depend on the characteristic of $K$.

If $\operatorname{char}(K)=5$, then $\mathcal{A}$ is free with exponents $[1,5,15]$. If $\operatorname{char}(K)=7$, then $\mathcal{A}$ is free with exponents $[1,7,13]$. If $\operatorname{char}(K) \neq 5,7$, then $\mathcal{A}$ is free with exponents $[1,9,11]$.

Let $\delta_{1}, \ldots, \delta_{l} \in D(\mathcal{A})$. Then $\operatorname{det}\left(\delta_{i}\left(x_{j}\right)\right)_{i, j}$ is divisible by $Q(\mathcal{A})$. One of the most famous characterization of freeness is due to Saito [14] and it uses the determinant of the coefficient matrix of $\delta_{1}, \ldots, \delta_{l}$ to check if the arrangement $\mathcal{A}$ is free or not. Notice that the original statement is for characteristic 0 , but in [17] Terao showed that this statement holds true for any characteristic.

Theorem 3.5 (Saito's criterion) Let $\mathcal{A}$ be a central arrangement in $K^{l}$ and consider $\delta_{1}, \ldots, \delta_{l} \in D(\mathcal{A})$. Then the following statements are equivalent 
1. $D(\mathcal{A})$ is free with basis $\delta_{1}, \ldots, \delta_{l}$, i. e. $D(\mathcal{A})=S \cdot \delta_{1} \oplus \cdots \oplus S \cdot \delta_{l}$.

2. $\operatorname{det}\left(\delta_{i}\left(x_{j}\right)\right)_{i, j}=c Q(\mathcal{A})$, where $c \in K \backslash\{0\}$.

3. $\delta_{1}, \ldots, \delta_{l}$ are linearly independent over $S$ and $\sum_{i=1}^{l} \operatorname{pdeg}\left(\delta_{i}\right)=n$.

Given an arrangement $\mathcal{A}$ in $K^{l}$, the Jacobian ideal of $\mathcal{A}$ is the ideal of $S$ generated by $Q(\mathcal{A})$ and all its partial derivatives, and it is denoted by $J(\mathcal{A})$. This ideal has a central role in the study of free arrangements. In fact, we can also characterize freeness by looking at the Jacobian ideal of $\mathcal{A}$. This characterization by Terao [15] will be used in Section 6 Notice that Terao described this result for characteristic 0 , but a similar proof (c.f. [10]) also works for any characteristic.

Theorem 3.6 (Terao's criterion) A central arrangement $\mathcal{A}$ in $K^{l}$ is free if and only if $S / J(\mathcal{A})$ is 0 or $(l-2)$-dimensional Cohen-Macaulay.

Proof If $\mathcal{A}$ has only one hyperplane, then $S / J(\mathcal{A})$ is 0 . Assume now that $|\mathcal{A}| \geq 2$. In this case $S / J(\mathcal{A}) \neq 0$ and $\operatorname{dim}(S / J(\mathcal{A}))=l-2$. Denote by $\mathcal{O}_{\mathcal{A}}$ the quotient $S /(Q(\mathcal{A})$. We have the exact sequence

$$
0 \longrightarrow D(\mathcal{A}) \stackrel{i}{\longrightarrow} S^{l} \stackrel{\beta}{\longrightarrow} J(\mathcal{A}) \mathcal{O}_{\mathcal{A}} \longrightarrow 0,
$$

where $i$ is the inclusion and

$$
\beta\left(\left(g_{1}, \ldots, g_{l}\right)^{t}\right)=\sum_{i=1}^{l} g_{i} \partial Q(\mathcal{A}) / \partial x_{i} \text { for }\left(g_{1}, \ldots, g_{l}\right)^{t} \in S^{l} .
$$

From the exact sequence (1) together with the Auslander-Buchsbaum equality (see [9]), we deduce that

$$
D(\mathcal{A}) \text { is free } \Leftrightarrow \operatorname{depth}\left(J(\mathcal{A}) \mathcal{O}_{\mathcal{A}}\right)=l-1 .
$$

The Depth Lemma (see [9]) applied now to the short exact sequence

$$
0 \longrightarrow J(\mathcal{A}) \mathcal{O}_{\mathcal{A}} \longrightarrow \mathcal{O}_{\mathcal{A}} \longrightarrow \mathcal{O}_{\mathcal{A}} / J(\mathcal{A}) \mathcal{O}_{\mathcal{A}} \longrightarrow 0
$$

shows that

$$
\operatorname{depth}\left(J(\mathcal{A}) \mathcal{O}_{\mathcal{A}}\right)=l-1 \Leftrightarrow \operatorname{depth}\left(\mathcal{O}_{\mathcal{A}} / J(\mathcal{A}) \mathcal{O}_{\mathcal{A}}\right)=l-2 .
$$

Since $S / J(\mathcal{A}) \cong \mathcal{O}_{\mathcal{A}} / J(\mathcal{A}) \mathcal{O}_{\mathcal{A}}$, we obtain that

$$
D(\mathcal{A}) \text { is free } \Leftrightarrow \operatorname{depth}(S / J(\mathcal{A}))=l-2 .
$$

Since we have the following equivalence

$\operatorname{depth}(S / J(\mathcal{A}))=l-2=\operatorname{dim}(S / J(\mathcal{A})) \Leftrightarrow S / J(\mathcal{A})$ is Cohen-Macaulay of dimension $l-2$, we obtain the thesis.

Freeness has several consequences. For example we recall the following. 
Theorem 3.7 ([16]) Suppose that $\mathcal{A}$ is a free arrangement in $K^{l}$ with exponents $\left(e_{1}, \ldots, e_{l}\right)$. Then

$$
\chi(\mathcal{A}, t)=\prod_{i=1}^{l}\left(t-e_{i}\right) .
$$

The previous theorem imposes a necessary condition on the structure of $L(\mathcal{A})$ for the arrangement $\mathcal{A}$ to be free. The Terao conjecture is the problem to ask the converse, i.e if the structure of $L(\mathcal{A})$ characterize freeness of $\mathcal{A}$. This conjecture is still unsolved. In [20], Yoshinaga gave an affirmative result when $K=\mathbb{F}_{p}$ and $l=3$. Specifically, he proved the following result.

Theorem $3.8([20])$ Let $\mathcal{A}$ be a central arrangement in $\mathbb{F}_{p}^{3}$. Then the following facts hold true.

1. When $|\mathcal{A}| \geq 2 p$, $\mathcal{A}$ is free if and only if $\chi(\mathcal{A}, p)=0$.

2. When $|\mathcal{A}|=2 p-1, \mathcal{A}$ is free if and only if either $\chi(\mathcal{A}, p)=0$ or $\chi(\mathcal{A}, t)=(t-1)(t-p+1)^{2}$.

3. When $|\mathcal{A}|=2 p-2$, $\mathcal{A}$ is free if and only if either $\chi(\mathcal{A}, p)=0$ or $\chi(\mathcal{A}, t)=(t-1)(t-p+1)(t-p+2)$.

More in general, Yoshinaga also proved the following.

Theorem $3.9([\mathbf{2 0}])$ Let $\mathcal{A}$ be a central arrangement in $\mathbb{F}_{p}^{l}$. If $\chi\left(\mathcal{A}, p^{l-2}\right)=0$, then $\mathcal{A}$ is free with exponents $\left(1, p, \ldots, p^{l-2},|\mathcal{A}|-1-p-\cdots-p^{l-2}\right)$.

\section{From characteristic 0 to characteristic $p$}

From now on we will assume that $\mathcal{A}=\left\{H_{1}, \ldots, H_{n}\right\}$ is a central arrangement in $\mathbb{Q}^{l}$. After clearing the denominators, we can suppose that $\alpha_{i} \in \mathbb{Z}\left[x_{1}, \ldots, x_{l}\right]$ for all $i=1, \ldots, n$, and hence that $Q(\mathcal{A})=\prod_{i=1}^{n} \alpha_{i} \in \mathbb{Z}\left[x_{1}, \ldots, x_{l}\right]$. Moreover, we can also assume that there exists no prime number $p$ that divides any $\alpha_{i}$.

Let $p$ be a prime number. Consider the image of $Q(\mathcal{A})$ under the canonical homomorphism $\pi_{p}: \mathbb{Z}\left[x_{1}, \ldots, x_{l}\right] \longrightarrow \mathbb{F}_{p}\left[x_{1}, \ldots, x_{l}\right]$.

Definition 4.1 Let $\mathcal{A}$ be a central arrangement in $\mathbb{Q}^{l}$. We will say that a prime number $p$ is $\operatorname{good}$ for $\mathcal{A}$ if $\pi_{p}(Q(\mathcal{A}))$ is reduced.

Lemma 4.2 There is a finite number of primes $p$ that are not good for $\mathcal{A}$.

Proof We have that $\pi_{p}(Q(\mathcal{A}))$ is not reduced if and only if $\pi_{p}\left(\alpha_{i}\right)$ is a multiple of $\pi_{p}\left(\alpha_{j}\right)$ for some $i \neq j$ and this can happen only for a finite number of primes.

Now let $p$ be a good prime for $\mathcal{A}$, and consider $\mathcal{A}_{p}$ the arrangement in $\mathbb{F}_{p}^{l}$ defined by $\pi_{p}(Q(\mathcal{A}))$. Hence, by construction, $Q\left(\mathcal{A}_{p}\right)=\pi_{p}(Q(\mathcal{A})) \neq 0$ and it is reduced.

Theorem 4.3 If $\mathcal{A}$ is free in $\mathbb{Q}^{l}$ with exponents $\left(e_{1}, \ldots, e_{l}\right)$, then $\mathcal{A}_{p}$ is free in $\mathbb{F}_{p}^{l}$ with exponents $\left(e_{1}, \ldots, e_{l}\right)$, for all good primes except possibly a finite number of them. 
Proof Let $\Delta=\left\{\delta_{1}, \ldots, \delta_{l}\right\}$ be a basis of the module $D(\mathcal{A})$ with $\operatorname{pdeg}\left(\delta_{i}\right)=e_{i}$, for all $i=1, \ldots, l$. After clearing all possible denominators, we can assume that every polynomial that appear in each $\delta \in \Delta$ is in $\mathbb{Z}\left[x_{1}, \ldots, x_{l}\right]$. Hence, if we write $\delta=\sum_{i=1}^{l} g_{i} \partial / \partial x_{i} \in \Delta$, we can consider $\bar{\delta}=\sum_{i=1}^{l} \pi_{p}\left(g_{i}\right) \partial / \partial x_{i} \in \operatorname{Der}_{\mathbb{F}_{p}^{l}}$. We can assume that $p \nmid \delta$ for all $\delta \in \Delta$, and hence $\bar{\delta} \neq 0$ for all $\delta \in \Delta$. This implies $\operatorname{pdeg}\left(\bar{\delta}_{i}\right)=\operatorname{pdeg}\left(\delta_{i}\right)=e_{i}$ for all $i=1, \ldots, l$.

By the definition of $D(\mathcal{A})$, for each $\delta=\sum_{i=1}^{l} g_{i} \partial / \partial x_{i} \in \Delta$ there exists $h \in$ $\mathbb{Z}\left[x_{1}, \ldots, x_{l}\right]$ such that $\delta(Q(\mathcal{A}))=h Q(\mathcal{A})$. If we apply $\pi_{p}$ to this expression, we obtain that $\bar{\delta}\left(Q\left(\mathcal{A}_{p}\right)\right)=\sum_{i=1}^{l} \pi_{p}\left(g_{i}\right) \partial \pi_{p}(Q(\mathcal{A})) / \partial x_{i}=\pi_{p}(\delta(Q(\mathcal{A})))=\pi_{p}(h Q(\mathcal{A}))=$ $\pi_{p}(h) \pi_{p}(Q(A))=\pi_{p}(h) Q\left(A_{p}\right)$, and hence $\bar{\delta} \in D\left(\mathcal{A}_{p}\right)$.

By Theorem 3.5 since in each $\delta \in \Delta$ every polynomial that appear have only integer coefficients and since there exists no prime number that divides any $\alpha_{i}$, there exists $c \in \mathbb{Z} \backslash\{0\}$ such that $\operatorname{det}\left(\delta_{i}\left(x_{j}\right)\right)_{i, j}=c Q(\mathcal{A})$. If we apply $\pi_{p}$ to the previous equality we obtain that $\operatorname{det}\left(\bar{\delta}_{i}\left(x_{j}\right)\right)_{i, j}=\pi_{p}\left(\operatorname{det}\left(\delta_{i}\left(x_{j}\right)\right)_{i, j}\right)=\pi_{p}(c Q(\mathcal{A}))=$ $\pi_{p}(c) Q\left(\mathcal{A}_{p}\right)$. Hence if $p$ does not divide $c$, we have $\pi_{p}(c) \in \mathbb{F}_{p} \backslash\{0\}$ and hence again by Theorem 3.5, we have that $\bar{\delta}_{1}, \ldots, \bar{\delta}_{l}$ are a basis of $D\left(\mathcal{A}_{p}\right)$. This proves that $\mathcal{A}_{p}$ is free with exponents $\left(e_{1}, \ldots, e_{l}\right)$.

By Lemma 4.2, the number of non-good primes is finite. Hence we have the following.

Corollary 4.4 Let $\mathcal{A}$ be a central arrangement in $\mathbb{Q}^{l}$ and $p$ a large prime number. If $\mathcal{A}$ is free in $\mathbb{Q}^{l}$ with exponents $\left(e_{1}, \ldots, e_{l}\right)$, then $\mathcal{A}_{p}$ is free in $\mathbb{F}_{p}^{l}$ with exponents $\left(e_{1}, \ldots, e_{l}\right)$.

From Example 3.3 , we have the following.

Example 4.5 Consider the arrangement $\mathcal{A}$ of Example 3.3 with $K=\mathbb{Q}$. Then the determinant of the coefficient matrix of the basis of $D(\mathcal{A})$ is equal to $2 Q(\mathcal{A})$. $A$ direct computation shows that if we take another basis of $D(\mathcal{A})$ with only integer coefficients, then the determinant of the coefficient matrix is equal to $c Q(\mathcal{A})$ with $c \in 2 \mathbb{Z} \backslash\{0\}$. This is why over $\mathbb{F}_{2}$ the exponents of $\mathcal{A}$ change.

Similarly to the previous example, we have the following

Example 4.6 Consider $\mathcal{A}$ the cone of $\mathcal{A}^{[-2,2]}$ the Shi-Catalan arrangement of type $B$ as in Example 3.4 with $K=\mathbb{Q}$. Then the determinant of the coefficient matrix of any basis of $D(\mathcal{A})$ with only integer coefficients is equal to $c Q(\mathcal{A})$, with $c \in 35 \mathbb{Z} \backslash\{0\}$. This is why over $\mathbb{F}_{5}$ and $\mathbb{F}_{7}$ the exponents of $\mathcal{A}$ change.

The following example shows that some good primes need indeed to be removed in Theorem 4.3. Specifically, it is an example of a free arrangements in $\mathbb{Q}^{l}$ that is not free in $\mathbb{F}_{p}^{l}$, for some good prime $p$.

Example 4.7 Consider $\mathcal{A}$ the arrangement in $\mathbb{Q}^{4}$ as the cone of $\mathcal{A}^{[-2,2]}$ the ShiCatalan arrangement of type $B$. As described in [2], $\mathcal{A}$ is a free arrangement with exponents $(1,13,15,17)$. Moreover, the determinant of the coefficient matrix of a basis of $D(\mathcal{A})$ with only integer coefficients is equal to $c Q(\mathcal{A})$, with $c \in 56595 \mathbb{Z} \backslash\{0\}$. Notice that $56595=3 \cdot 5 \cdot 7^{3} \cdot 11$. Now, 3 is not a good prime but 5, 7 and 11 are. A direct computation shows that the arrangement $\mathcal{A}_{5}$ over $\mathbb{F}_{5}$ is free with exponents $(1,5,15,25)$. However, both $\mathcal{A}_{7}$ over $\mathbb{F}_{7}$ and $\mathcal{A}_{11}$ over $\mathbb{F}_{11}$ are not free. 
5 Homogeneous ideals in $\mathbb{Z}\left[x_{1}, \ldots, x_{l}\right]$

In this section, we study ideals in $\mathbb{Z}\left[x_{1}, \ldots, x_{l}\right]$, their Betti numbers and their zero divisors. This will play an important role in Section 6 .

Definition 5.1 Given a sequence of numbers $\left\{c_{i, j}\right\}_{i, j \in \mathbb{N}}$, we obtain a new sequence by a cancellation as follows. Fix a $j$, and choose $i$ and $i^{\prime}$ so that one of the numbers is odd and the other is even. Then replace $c_{i, j}$ by $c_{i, j}-1$, and replace $c_{i^{\prime}, j}$ by $c_{i^{\prime}, j}-1$. We have a consecutive cancellation when $i^{\prime}=i+1$.

The following result is a generalization of Proposition 5.3 from the first arXiv version of [8] and it will play an important role in Theorem 6.1.

Proposition 5.2 Let $I$ be a homogeneous ideal of the ring $R=\mathbb{Z}\left[x_{1}, \ldots, x_{l}\right]$ and $p$ a prime number that is a non-zero divisor in $R / I$. Then, the graded Betti numbers of $(R / I) \otimes_{\mathbb{Z}} \mathbb{Q}$ can be obtained from the ones of $(R / I) \otimes_{\mathbb{Z}} \mathbb{F}_{p}$ by a sequence of consecutive cancellations.

Proof Since $p$ is a non-zero divisor in $R / I$, then $(R / I) \otimes_{\mathbb{Z}} \mathbb{Z}_{(p)}$ is flat over $\mathbb{Z}_{(p)}$. Let $\mathbb{H}_{\bullet}$ be a minimal graded free $R \otimes_{\mathbb{Z}} \mathbb{Z}_{(p)}$-resolution of $(R / I) \otimes_{\mathbb{Z}} \mathbb{Z}_{(p)}$. The minimality assumption on $\mathbb{H}$. means that we can choose homogeneous bases for the free modules in this complex such that the entries in the matrices are homogeneous and they belong to the ideal $\left(p, x_{1}, \ldots, x_{l}\right)$. This implies that $\mathbb{H}_{\bullet} \otimes_{\mathbb{Z}} \mathbb{Z}_{(p)}$ is a free resolution of $(R / I) \otimes_{\mathbb{Z}} \mathbb{Z}_{(p)}$ over $R \otimes_{\mathbb{Z}} \mathbb{Z}_{(p)}=\mathbb{Z}_{(p)}\left[x_{1}, \ldots, x_{l}\right]$, and that the graded Betti numbers of $\mathbb{H}_{\bullet}$ and $\mathbb{H}_{\bullet} \otimes_{\mathbb{Z}} \mathbb{Z}_{(p)}$ are the same. The flatness of $(R / I) \otimes_{\mathbb{Z}} \mathbb{Z}_{(p)}$ over $\mathbb{Z}_{(p)}$ ensures that the complex $\mathbb{H}_{\bullet} \otimes_{\mathbb{Z}} \mathbb{F}_{p}=\mathbb{H}_{\bullet} \otimes_{\mathbb{Z}} \mathbb{Z}_{(p)} \otimes_{\mathbb{Z}_{(p)}} \mathbb{F}_{p}$ is a free resolution of $(R / I) \otimes_{\mathbb{Z}} \mathbb{F}_{p}=(R / I) \otimes_{\mathbb{Z}} \mathbb{Z}_{(p)} \otimes_{\mathbb{Z}_{(p)}} \mathbb{F}_{p}$ over $R \otimes_{\mathbb{Z}} \mathbb{F}_{p}=\mathbb{F}_{p}\left[x_{1}, \ldots, x_{l}\right]$. Now matrices giving the maps in this complex are in $\left(x_{1}, \ldots, x_{l}\right)$, i.e., it is a minimal resolution. This shows that the graded Betti number of $\mathbb{H}_{\bullet}$ coincides with the one of $(R / I) \otimes_{\mathbb{Z}} \mathbb{F}_{p}$.

Now $\mathbb{H}_{\bullet} \otimes_{\mathbb{Z}_{(p)}} \mathbb{Q}$ is a graded free $\left(R \otimes_{\mathbb{Z}} \mathbb{Q}\right)$-resolution of $(R / I) \otimes_{\mathbb{Z}} \mathbb{Q}$. Therefore we can write $\mathbb{H}_{\bullet} \otimes_{\mathbb{Z}_{(p)}} \mathbb{Q}=\mathbb{G}_{\bullet} \oplus \mathbb{G}_{\bullet}^{\prime}$, where $\mathbb{G}_{\bullet}$ is a minimal graded free $\left(R \otimes_{\mathbb{Z}} \mathbb{Q}\right)$ resolution of $(R / I) \otimes_{\mathbb{Z}} \mathbb{Q}$ and $\mathbb{G}_{\bullet}^{\prime}$ is a graded trivial complex of free $\left(R \otimes_{\mathbb{Z}} \mathbb{Q}\right)$ modules [9, Theorem 20.2]. Therefore, the graded Betti numbers of $(R / I) \otimes_{\mathbb{Z}} \mathbb{Q}$ can be obtained from the one of $(R / I) \otimes_{\mathbb{Z}} \mathbb{F}_{p}$ by a sequence of consecutive cancellations.

From the Proposition 5.2, we directly obtain the relation between projective dimensions.

Corollary 5.3 Let I be a homogeneous ideal of $R=\mathbb{Z}\left[x_{1}, \ldots, x_{l}\right]$ and $p$ a prime number that is a non-zero divisor in $R / I$. Then, the Betti numbers of $(R / I) \otimes_{\mathbb{Z}} \mathbb{Q}$ are smaller or equal to the one of $(R / I) \otimes_{\mathbb{Z}} \mathbb{F}_{p}$, and hence $\operatorname{pdim}\left((R / I) \otimes_{\mathbb{Z}} \mathbb{Q}\right) \leq$ $\operatorname{pdim}\left((R / I) \otimes_{\mathbb{Z}} \mathbb{F}_{p}\right)$.

In general, given $I$ an ideal of $R=\mathbb{Z}\left[x_{1}, \ldots, x_{l}\right]$, the number of zero divisor in $R / I$ is infinite. However, if we restrict our attention to zero divisors that are prime numbers, we have the following.

Proposition 5.4 Let $I$ be an ideal of $R=\mathbb{Z}\left[x_{1}, \ldots, x_{l}\right]$. Then the number of distinct prime numbers that are zero divisors in $R / I$ is finite. 
Proof By Theorem 14.4 of $[9]$, there exists $a \in \mathbb{Z} \backslash\{0\}$ such that $(R / I)\left[a^{-1}\right]$ is a free $\mathbb{Z}\left[a^{-1}\right]$-module. This implies that the set of distinct prime numbers that are zero divisors in $R / I$ is included in the set of distinct prime numbers that divide $a$, that is finite by the unique factorization theorem.

As described in Proposition 5.4 the cardinality of prime numbers that are zero divisors in $R / I$ is finite. The rest of this section is dedicated to describe a method to compute them via the theory of Gröbner basis.

Consider $I$ an ideal of $R=\mathbb{Z}\left[x_{1}, \ldots, x_{l}\right]$ and $\sigma$ a term ordering on $R$. As for the case of polynomial rings over fields, we can study the theory of Gröbner basis for ideals of $R$. We refer to Chapter 4.5 of [4] for details.

Given $f \in R$, we define the leading term of $f$ as $\operatorname{LT}_{\sigma}(f)=\max _{\sigma}\{t \in$ $\operatorname{Supp}(f)\}$, the leading coefficient of $f$ as the coefficient multiplying the $\operatorname{LT}_{\sigma}(f)$ in the writing of $f$ and we denote it by $\mathrm{LC}_{\sigma}(f)$, and the leading monomial of $f$ as $\operatorname{LM}_{\sigma}(f)=\mathrm{LC}_{\sigma}(f) \operatorname{LT}_{\sigma}(f)$.

Definition 5.5 Let $I$ be an ideal of $R=\mathbb{Z}\left[x_{1}, \ldots, x_{l}\right]$, $\sigma$ a term ordering on $R$ and $G=\left\{g_{1}, \ldots, g_{t}\right\}$ a set of non-zero polynomials in $I$. Then we say that $G$ is a minimal strong $\sigma$-Gröbner basis for I if the following conditions hold true

1. G forms a set of generators of $I$;

2. for each $f \in I$, there exists $i \in\{1, \ldots, t\}$ such that $\mathrm{LM}_{\sigma}\left(g_{i}\right)$ divides $\mathrm{LM}_{\sigma}(f)$;

3. if $i \neq j$, then $\operatorname{LM}_{\sigma}\left(g_{i}\right)$ does not divide $\operatorname{LM}_{\sigma}\left(g_{j}\right)$.

Remark 5.6 (c.f. [4], Lemma 4.5.8) The reduced $\sigma$-Gröbner basis of an ideal I of $\mathbb{Z}\left[x_{1}, \ldots, x_{l}\right]$ is also a minimal strong $\sigma$-Gröbner basis of I. Moreover, every minimal strong $\sigma$-Gröbner basis of $I$ is also a $\sigma$-Gröbner basis.

Proposition 5.7 ([4], Exercise 4.5.9) Let I be a non-zero ideal of $R=\mathbb{Z}\left[x_{1}, \ldots, x_{l}\right]$ and $\sigma$ a term ordering on $R$. Then there always exists a minimal strong $\sigma$-Gröbner basis of $I$

Notice that in general a minimal strong $\sigma$-Gröbner basis is not unique. Consider the following example.

Example 5.8 Consider the ideal $I=\left(x^{2}-y, 3 y\right)$ in $\mathbb{Z}[x, y]$ and $\sigma=$ degrevlex. Then both $\left\{x^{2}-y, 3 y\right\}$ and $\left\{x^{2}+2 y, 3 y\right\}$ are minimal strong $\sigma$-Gröbner basis for $I$.

Lemma 5.9 Let $I$ be an ideal of the polynomial ring $R=\mathbb{Z}\left[x_{1}, \ldots, x_{l}\right]$, and $\sigma$ a term ordering on $R$. Let $G_{1}$ and $G_{2}$ be two minimal strong $\sigma$-Gröbner bases of $I$. Then $\left\{\mathrm{LM}_{\sigma}(g) \mid g \in G_{1}\right\}=\left\{\mathrm{LM}_{\sigma}(g) \mid g \in G_{2}\right\}$. Consequently we have $\left|G_{1}\right|=\left|G_{2}\right|$ and $\left\{\mathrm{LC}_{\sigma}(g) \mid g \in G_{1}\right\}=\left\{\operatorname{LC}_{\sigma}(g) \mid g \in G_{2}\right\}$.

Proof Assume that there exists $t \in \mathrm{LM}_{\sigma}\left(G_{1}\right) \backslash \mathrm{LM}_{\sigma}\left(G_{2}\right)$. Now, $t \in \mathrm{LM}_{\sigma}(I)$ and hence there exists $f \in I$ such that $t=\operatorname{LM}_{\sigma}(f)$. Since $G_{2}$ is a minimal strong $\sigma$ Gröbner basis for $I$, there exists $g \in G_{2}$ such that $\operatorname{LM}_{\sigma}(g)=t^{\prime}$ divides $\operatorname{LM}_{\sigma}(f)=t$. With a similar argument, we see that $t^{\prime}$, and hence $t$, is a multiple of an element $t^{\prime \prime}$ in $\operatorname{LM}_{\sigma}\left(G_{1}\right)$. The minimality of $G_{1}$ implies that $t^{\prime \prime}=t$. Hence, $t=t^{\prime} \in \operatorname{LM}_{\sigma}\left(G_{2}\right)$, but this is a contradiction. This shows that $\operatorname{LM}_{\sigma}\left(G_{1}\right) \subseteq \operatorname{LM}_{\sigma}\left(G_{2}\right)$.

With the same argument we can show that $\operatorname{LM}_{\sigma}\left(G_{2}\right) \subseteq \operatorname{LM}_{\sigma}\left(G_{1}\right)$, and hence that $\operatorname{LM}_{\sigma}\left(G_{1}\right)=\operatorname{LM}_{\sigma}\left(G_{2}\right)$. 
Remark 5.10 The previous lemma implies that $\left\{\mathrm{LM}_{\sigma}(g) \mid g \in G\right\}$ generates the monomial ideal $\mathrm{LM}_{\sigma}(I)$. details.

By Lemma 5.9, we can introduce the following definition. See [13], for more

Definition 5.11 Let $I$ be an ideal of $R=\mathbb{Z}\left[x_{1}, \ldots, x_{l}\right]$, and $\sigma$ be a term ordering on $R$. If a prime number $p$ does not divide the leading coefficient of any polynomial in a minimal strong $\sigma$-Gröbner basis for $I$, then we will say $p$ is $\sigma$-lucky for $I$.

Remark 5.12 Given $I$ an ideal of $R=\mathbb{Z}\left[x_{1}, \ldots, x_{l}\right]$ and $\sigma$ a term ordering on $R$, since a minimal strong $\sigma$-Gröbner basis is finite, then the number of primes that are not $\sigma$-lucky for I is finite.

Notice that the list of $\sigma$-lucky primes for an ideal depends on the choice of term ordering.

Example 5.13 Consider the ideal $I=(3 x-y)$ in $\mathbb{Z}[x, y]$. Consider on $\mathbb{Z}[x, y] a$ term ordering $\sigma$ such that $x>_{\sigma} y$. Then $\{3 x-y\}$ is a minimal strong $\sigma$-Gröbner basis of $I$, and hence every prime $p \neq 3$ is $\sigma$-lucky for $I$. However, if we consider on $\mathbb{Z}[x, y]$ a term ordering $\tau$ such that $y>_{\tau} x$, then $\{y-3 x\}$ is a minimal strong $\tau$-Gröbner basis of I. Hence every prime $p$ is $\tau$-lucky for $I$, also $p=3$.

Notice that in general, we cannot determine the $\sigma$-lucky primes from the coefficients that appear in a set of generators.

Example 5.14 Consider the ideal $I=(2 x+3 y, x-y)$ in $\mathbb{Z}[x, y]$ with $\sigma=$ degrevlex. In this case, $\{5 y, x-y\}$ is a minimal strong $\sigma$-Gröbner basis of $I$, and hence $p=5$ is the only non $\sigma$-lucky prime of $I$.

We are now ready to describe the relation between $\sigma$-lucky primes and primes that are non-zero divisors.

Proposition 5.15 Let $I$ be an ideal of $R=\mathbb{Z}\left[x_{1}, \ldots, x_{l}\right]$ and $\sigma$ a term ordering on $R$. If $p$ is a $\sigma$-lucky prime for $I$, then $p$ is a non-zero divisor in $R / I$.

Proof Let $G=\left\{g_{1}, \ldots, g_{t}\right\}$ be a minimal strong $\sigma$-Gröbner basis of $I$ and $p$ a $\sigma$ lucky prime for $I$. Suppose that $p$ is a zero divisor in $R / I$. This implies that there exists $f \in R \backslash I$ such that $p f \in I$. By definition of minimal strong $\sigma$-Gröbner basis, there exists $i \in\{1, \ldots, t\}$ such that $\mathrm{LM}_{\sigma}\left(g_{i}\right)$ divides $\mathrm{LM}_{\sigma}(p f)$. We can now consider $h_{1}=p f-\frac{\mathrm{LM}_{\sigma}(p f)}{\mathrm{LM}_{\sigma}\left(g_{i}\right)} g_{i} \in I$. Since $p$ is $\sigma$-lucky, $p \mid \frac{\mathrm{LM}_{\sigma}(p f)}{\mathrm{LM}_{\sigma}\left(g_{j}\right)}$, and hence we have $h_{1}=p f_{1}$, for some $f_{1} \in R$. We can now repeat the process with $p f_{1} \in I$ and obtain $p f_{2}=p f_{1}-\frac{\mathrm{LM}_{\sigma}\left(p f_{1}\right)}{\mathrm{LM}_{\sigma}\left(g_{j}\right)} g_{j} \in I$, for some $j$. Since $G$ is a $\sigma$-Gröbner basis this process will end after a finite number of steps, i.e. we will obtain that there exists a $k \geq 1$ such that $p f_{k} \in I$ with $p f_{k} \neq 0$ but $p f_{k+1}=0$. Retrieving each step, we can write $p f=\sum_{i=1}^{t} p a_{i} g_{i}$, for some $a_{i} \in R$. This implies that $f=\sum_{i=1}^{t} a_{i} g_{i} \in I$, but this is impossible by assumption.

Notice that in general the set of distinct prime numbers that are non-zero divisors in $R / I$ contains strictly the set of $\sigma$-lucky primes for $I$.

Example 5.16 Consider the ideal of Example 5.13 with the term ordering $\sigma$. Then $p=3$ is a non-zero divisor in $R / I$, but it is not a $\sigma$-lucky prime. 


\section{From characteristic $p$ to characteristic 0}

As in Section 4, we will assume that $\mathcal{A}=\left\{H_{1}, \ldots, H_{n}\right\}$ is a central arrangement in $\mathbb{Q}^{l}$ and hence that $\alpha_{i} \in \mathbb{Z}\left[x_{1}, \ldots, x_{l}\right]$ for all $i=1, \ldots, n$. Moreover, we can also assume that there exists no prime number $p$ that divides any $\alpha_{i}$.

In the rest of the paper, we will denote by $J(\mathcal{A})_{\mathbb{Z}}$ the ideal of $\mathbb{Z}\left[x_{1}, \ldots, x_{l}\right]$ generated by $Q(\mathcal{A})$ and its partial derivatives.

Theorem 6.1 Let $\mathcal{A}=\left\{H_{1}, \ldots, H_{n}\right\}$ be a central arrangement in $\mathbb{Q}^{l}$. Let $p$ be a good prime number for $\mathcal{A}$ that is a non-zero divisor in $\mathbb{Z}\left[x_{1}, \ldots, x_{l}\right] / J(\mathcal{A})_{\mathbb{Z}}$. If $\mathcal{A}_{p}$ is free in $\mathbb{F}_{p}^{l}$ with exponents $\left(e_{1}, \ldots, e_{l}\right)$, then $\mathcal{A}$ is free in $\mathbb{Q}^{l}$ with exponents $\left(e_{1}, \ldots, e_{l}\right)$.

Proof Denote $S=\mathbb{Q}\left[x_{1}, \ldots, x_{l}\right], S_{p}=\mathbb{F}_{p}\left[x_{1}, \ldots, x_{l}\right]$ and $R=\mathbb{Z}\left[x_{1}, \ldots, x_{l}\right]$. Notice that $S_{p} / J\left(\mathcal{A}_{p}\right) \cong\left(R / J(\mathcal{A})_{\mathbb{Z}}\right) \otimes_{\mathbb{Z}} \mathbb{F}_{p}$ and $S / J(\mathcal{A}) \cong\left(R / J(\mathcal{A})_{\mathbb{Z}}\right) \otimes_{\mathbb{Z}} \mathbb{Q}$.

If $n=1$, then both $S_{p} / J\left(\mathcal{A}_{p}\right)$ and $S / J(\mathcal{A})$ are zero.

Assume now that $n \geq 2$. This implies that $S_{p} / J\left(\mathcal{A}_{p}\right) \neq 0$. By Theorem 3.6 $S_{p} / J\left(\mathcal{A}_{p}\right)$ is Cohen-Macaulay of dimension $l-2$. By Auslander-Buchsbaum equality, we can write

$\operatorname{depth}\left(S_{p} / J\left(\mathcal{A}_{p}\right)\right)+\operatorname{pdim}\left(S_{p} / J\left(\mathcal{A}_{p}\right)\right)=l=\operatorname{depth}(S / J(\mathcal{A}))+\operatorname{pdim}(S / J(\mathcal{A}))$.

By Corollary 5.3 $\operatorname{pdim}\left(S_{p} / J\left(\mathcal{A}_{p}\right)\right) \geq \operatorname{pdim}(S / J(\mathcal{A}))$ and hence, by the previous equality, $\operatorname{depth}\left(S_{p} / J\left(\mathcal{A}_{p}\right)\right) \leq \operatorname{depth}(S / J(\mathcal{A}))$. Now $S_{p} / J\left(\mathcal{A}_{p}\right)$ is Cohen-Macaulay, hence $\operatorname{depth}\left(S_{p} / J\left(\mathcal{A}_{p}\right)\right)=\operatorname{dim}\left(S_{p} / J\left(\mathcal{A}_{p}\right)\right)=l-2=\operatorname{dim}(S / J(\mathcal{A}))$. This implies that $\operatorname{depth}(S / J(\mathcal{A})) \geq l-2=\operatorname{dim}(S / J(\mathcal{A}))$. On the other hand, $\operatorname{depth}(S / J(\mathcal{A})) \leq$ $\operatorname{dim}(S / J(\mathcal{A}))$ and hence we have that $\operatorname{depth}(S / J(\mathcal{A}))=\operatorname{dim}(S / J(\mathcal{A}))$. This implies that $S / J(\mathcal{A})$ is Cohen-Macaulay of dimension $l-2$, and so $\mathcal{A}$ is free in $\mathbb{Q}^{l}$.

This argument together with equation (1) show that $2=\operatorname{pdim}\left(S_{p} / J\left(\mathcal{A}_{p}\right)\right)=$ $\operatorname{pdim}(S / J(\mathcal{A}))$, and hence, $S / J(\mathcal{A})$ have the same Betti numbers as $S_{p} / J\left(\mathcal{A}_{p}\right)$. Since the exponents of $\mathcal{A}$ can be computed uniquely from the Betti numbers of $S / J(\mathcal{A})$, this implies that $\mathcal{A}$ has exponents $\left(e_{1}, \ldots, e_{l}\right)$.

In Theorem 6.1 the assumption that the prime $p$ is a non-zero divisor in the quotient $\mathbb{Z}\left[x_{1}, \ldots, x_{l}\right] / J(\mathcal{A})_{\mathbb{Z}}$ is fundamental. In fact we have the following.

Example 6.2 Consider the arrangement $\mathcal{A} \subseteq \mathbb{Q}^{3}$ with defining polynomial $Q(\mathcal{A})=$ $z(x+2 y-4 z)(y+4 z)(x+3 y-6 z)$. $\mathcal{A}$ is non free and both 2 and 3 are zero divisors in $\mathbb{Z}\left[x_{1}, \ldots, x_{l}\right] / J(\mathcal{A})_{\mathbb{Z}}$. In fact, we have that $3\left(y^{2} z^{2}+2 y z^{3}-8 z^{4}\right) \in J(\mathcal{A})_{\mathbb{Z}}$ but $y^{2} z^{2}+2 y z^{3}-8 z^{4} \notin J(\mathcal{A})_{\mathbb{Z}}$, and similarly $2\left(x y z^{2}+4 y^{2} z^{2}+4 x z^{3}+8 y z^{3}-32 z^{4}\right) \in$ $J(\mathcal{A})_{\mathbb{Z}}$ but $x y z^{2}+4 y^{2} z^{2}+4 x z^{3}+8 y z^{3}-32 z^{4} \notin J(\mathcal{A})_{\mathbb{Z}}$. However, both $A_{2}$ and $A_{3}$ are free with exponents $(1,1,2)$.

By Proposition 5.15, if $\sigma$ is a term ordering on $\mathbb{Z}\left[x_{1}, \ldots, x_{l}\right]$, then every $\sigma$-lucky prime for $J(\mathcal{A})_{\mathbb{Z}}$ is also a non-zero divisor in $\mathbb{Z}\left[x_{1}, \ldots, x_{l}\right] / J(\mathcal{A})_{\mathbb{Z}}$. This fact together with Theorem 6.1 gives us the following corollary.

Corollary 6.3 Let $\mathcal{A}$ be a central arrangement in $\mathbb{Q}^{l}$. Let $p$ be a good prime number for $\mathcal{A}$ that is a $\sigma$-lucky prime for $J(\mathcal{A})_{\mathbb{Z}}$, for some term ordering $\sigma$. If $\mathcal{A}_{p}$ is free in $\mathbb{F}_{p}^{l}$ with exponents $\left(e_{1}, \ldots, e_{l}\right)$, then $\mathcal{A}$ is free in $\mathbb{Q}^{l}$ with exponents $\left(e_{1}, \ldots, e_{l}\right)$. 
By Proposition 5.4, the cardinality of prime numbers that are zero divisors in the quotient $\mathbb{Z}\left[x_{1}, \ldots, x_{l}\right] / J(\mathcal{A})_{\mathbb{Z}}$ is finite. Hence, putting together Corollary 4.4 and Theorem 6.1, we have the following.

Corollary 6.4 Let $\mathcal{A}$ be a central arrangement in $\mathbb{Q}^{l}$ and p a large prime number. $\mathcal{A}_{p}$ is free in $\mathbb{F}_{p}^{l}$ with exponents $\left(e_{1}, \ldots, e_{l}\right)$ if and only if $\mathcal{A}$ is free in $\mathbb{Q}^{l}$ with exponents $\left(e_{1}, \ldots, e_{l}\right)$.

From Theorems 3.8 and 6.1 , we have the following.

Theorem 6.5 Let $\mathcal{A}$ be a central arrangement in $\mathbb{Q}^{3}$. Let $p$ be a good prime number for $\mathcal{A}$ that is a non-zero divisor in $\mathbb{Z}\left[x_{1}, x_{2}, x_{3}\right] / J(\mathcal{A})_{\mathbb{Z}}$. Then the following facts hold true.

1. When $|\mathcal{A}| \geq 2 p$, $\mathcal{A}$ is free if $\chi\left(\mathcal{A}_{p}, p\right)=0$.

2. When $|\mathcal{A}|=2 p-1, \mathcal{A}$ is free if either $\chi\left(\mathcal{A}_{p}, p\right)=0$ or $\chi\left(\mathcal{A}_{p}, t\right)=(t-1)(t-p+1)^{2}$.

3. When $|\mathcal{A}|=2 p-2$, $\mathcal{A}$ is free if either $\chi\left(\mathcal{A}_{p}, p\right)=0$ or $\chi\left(\mathcal{A}_{p}, t\right)=(t-1)(t-p+1)(t-p+2)$.

More in general, from Theorems 3.9 and 6.1, we have the following.

Theorem 6.6 Let $\mathcal{A}$ be a central arrangement in $\mathbb{Q}^{l}$. Let $p$ be a good prime number for $\mathcal{A}$ that is a non-zero divisor in $\mathbb{Z}\left[x_{1}, \ldots, x_{l}\right] / J(\mathcal{A})_{\mathbb{Z}}$. If $\chi\left(\mathcal{A}_{p}, p^{l-2}\right)=0$, then $\mathcal{A}$ is a free arrangement with exponents $\left(1, p, \ldots, p^{l-2},|\mathcal{A}|-1-p-\cdots-p^{l-2}\right)$.

As noted in [5], if $p$ is a large prime number, then $\mathcal{A}$ and $\mathcal{A}_{p}$ have isomorphic intersection lattices, and hence have the same characteristic polynomial. This fact, together with the finiteness of good prime numbers that are zero divisors in $\mathbb{Z}\left[x_{1}, \ldots, x_{l}\right] / J(\mathcal{A})_{\mathbb{Z}}$, allows us to say that if $\mathcal{A}$ is a central arrangement in $\mathbb{Q}^{l}$, $p$ is a large prime number, and $\chi\left(\mathcal{A}, p^{l-2}\right)=0$, then $\mathcal{A}$ is free with exponents $\left(1, p, \ldots, p^{l-2},|\mathcal{A}|-1-p-\cdots-p^{l-2}\right)$.

Example 6.7 Consider the arrangement $\mathcal{A}$ in $\mathbb{Q}^{3}$ with defining equation $Q(\mathcal{A})=$ $x y z(x-y)(x+y)(x-z)(x+z)(y-z)(y+z)$. Now, $p=5$ is a good prime number for $\mathcal{A}$ that does not divide $|\mathcal{A}|=9$ and that is a non-zero divisor in $\mathbb{Z}[x, y, z] / J(\mathcal{A})_{\mathbb{Z}}$. A direct computations shows that, $\chi\left(\mathcal{A}_{5}, t\right)=(t-1)(t-3)(t-5)$ and hence, by Theorem 6.5 or 6.6. $\mathcal{A}$ is free with exponents $(1,3,5)$. Notice that in this case, $\mathcal{A}$ and $\mathcal{A}_{5}$ have isomorphic intersection lattice, hence $p=5$ is a "large prime number". However, in general, it is difficult to detect when a prime number is "large" enough.

Acknowledgements The authors would like to thank G. Caviglia, M. Kummini, N. Nakashima and M. Yoshinaga for many helpful discussions.

\section{References}

1. Abe, T.: Divisionally free arrangements of hyperplanes. Inventiones mathematicae 204(1), 317-346 (2016). DOI 10.1007/s00222-015-0615-7

2. Abe, T., Terao, H.: The freeness of Shi-Catalan arrangements. European Journal of Combinatorics 32(8), 1191-1198 (2011) 
3. Abe, T., Yoshinaga, M.: Free arrangements and coefficients of characteristic polynomials. Mathematische Zeitschrift 275(3-4), 911-919 (2013)

4. Adams, W.W., Loustaunau, P.: An introduction to Gröbner bases. 3. American Mathematical Soc. (1994)

5. Ardila, F.: Computing the Tutte polynomial of a hyperplane arragement. Pacific Journal of Mathematics 230(1), 1-26 (2007)

6. Bigatti, A., Palezzato, E., Torielli, M.: New characterizations of freeness for hyperplane arrangements arXiv:1801.09868. To appear in Journal of Algebraic Combinatorics (2018)

7. Crapo, H., Rota, G.C.: On the foundations of combinatorial theory: Combinatorial geometries. MIT press Cambridge, Mass. (1970)

8. Dalili, K., Kummini, M.: Dependence of Betti numbers on characteristic. Communications in Algebra 42(2), 563-570 (2014)

9. Eisenbud, D.: Commutative algebra with a view toward algebraic geometry, vol. 150. Springer (1995)

10. Mond, D.: Notes on logarithmic vector fields, logarithmic differential forms and free divisors. Preprint (2012)

11. Orlik, P., Solomon, L.: Combinatorics and topology of complements of hyperplanes. Inventiones mathematicae 56(2), 167-189 (1980)

12. Orlik, P., Terao, H.: Arrangements of hyperplanes, Grundlehren der Mathematischen Wissenschaften [Fundamental Principles of Mathematical Sciences], vol. 300. Springer-Verlag, Berlin (1992)

13. Pauer, F.: On lucky ideals for Gröbner basis computations. Journal of Symbolic Computation 14(5), 471-482 (1992)

14. Saito, K.: Theory of logarithmic differential forms and logarithmic vector fields. J. Fac. Sci. Univ. Tokyo Sect. IA Math. 27(2), 265-291 (1980)

15. Terao, H.: Arrangements of hyperplanes and their freeness I. J. Fac. Sci. Univ. Tokyo Sect. IA Math. 27(2), 293-312 (1980)

16. Terao, H.: Generalized exponents of a free arrangement of hyperplanes and Shepherd-Todd-Brieskorn formula. Inventiones mathematicae 63(1), 159-179 (1981)

17. Terao, H.: Free arrangements of hyperplanes over an arbitrary field. Proceedings of the Japan Academy, Series A, Mathematical Sciences 59(7), 301-303 (1983)

18. Yoshinaga, M.: Characterization of a free arrangement and conjecture of Edelman and Reiner. Inventiones mathematicae 157(2), 449-454 (2004)

19. Yoshinaga, M.: On the freeness of 3-arrangements. Bulletin of the London Mathematical Society 37(01), 126-134 (2005)

20. Yoshinaga, M.: Free arrangements over finite field. Proceedings of the Japan Academy, Series A, Mathematical Sciences 82(10), 179-182 (2007)

21. Zaslavsky, T.: Facing up to Arrangements: Face-Count Formulas for Partitions of Space by Hyperplanes, vol. 154. Memoirs American Mathematical Society (1975) 